\title{
Volition and Imagery in Neurorehabilitation
}

\author{
Martin Lotze, MD* and Leonardo G. Cohen, $M D \dagger$
}

\begin{abstract}
New interventional approaches have been proposed in the last few years to treat the motor deficits resulting from brain lesions. Training protocols represent the gold-standard of these approaches. However, the degree of motor recovery experienced by most patients remains incomplete. It would be important to improve our understanding of the mechanisms underlying functional recovery. This chapter examines the role of two possible mechanisms that could operate to improve motor function in this setting: volition and motor imagery. It is argued that both represent possible strategies to enhance training effects.
\end{abstract}

Key Words: motivation, attention, motor imagery, hemiparesis, rehabilitation, forced use, volition, stroke, motor system, motor cortex

(Cog Behav Neurol 2006;19:135-140)

$M$ otor training protocols represent one of the fundamental bases of rehabilitative treatments after brain damage. Different strategies have been proposed to enhance training effects, including enhanced motivation, focused attention, motor imagery, progressive transfer of tasks towards the paretic limb, forced use, and integration of multimodal and emotional settings. The neural mechanisms underlying these strategies are poorly understood. In this review, we describe studies performed to better understand the influence of some of these factors on performance improvements and changes in intracortical excitatory and inhibitory mechanisms in humans undergoing training protocols. Focus is on the role of (a) volition in motor learning, (b) imagery in neurorehabilitation and motor control, and (c) neural substrates underlying performance of simple and complex movements after stroke.

Received for publication November 14, 2005; accepted November 20, 2005.

From the *Institute of Medical Psychology and Behavioral Neurobio logy, University of Tuebingen, Germany; and †Human Cortical Physiology Section, National Institute of Neurological Disorders and Stroke, National Institutes of Health, Bethesda.

Reprints: Martin Lotze, MD, Institut für Medizinische Psychologie und Verhaltensneurobiologie, Eberhard-Karls-Universität Tübingen, Gartenstraße 29, D-72074 Tübingen, Germany (e-mail: martin. lotze@uni-tuebingen.de).

Copyright (C) 2006 by Lippincott Williams \& Wilkins

\section{ROLE OF VOLITION IN MOTOR LEARNING}

Motor training protocols in patients with brain lesions elicit well-described changes in brain organization and performance improvements. ${ }^{1-6}$ One limitation of training protocols is that patients with more profound weakness are unable to carry out the motor routines required. The finding that passively elicited motions lead to activation and cortical reorganization in brain regions common to those activated with performance of voluntary movements suggested that it could also elicit improvements in motor function. ${ }^{7,8}$ This proposal has important implications for the design of neurorehabilitative treatments after stroke, particularly in patients who are too weak to perform effective voluntary motor training.

One recent study compared behavioral gains after short-term motor learning, changes in functional magnetic resonance imaging (fMRI) activation in the contralateral primary motor cortex (cM1) and in motor cortex excitability measured with transcranial magnetic stimulation (TMS) after a 30 minute training period consisting of either voluntarily or passively induced wrist movements in 2 different sessions in healthy volunteers. ${ }^{9}$ During active training, subjects were instructed to perform voluntary wrist flexion-extension movements of a specified duration in an articulated splint. Therefore, voluntary movements falling within a specified time window displayed on the screen monitor were considered correct hits. Subjects received a feedback signal after each training movement and hits were rewarded. If the movement's range was not complete, the program presented a negative feedback signal ("no movement performed"). Passive training consisted of wrist flexionextension movements of the same amplitude and duration range as in the active task elicited by a torque motor. fMRI activation and TMS parameters of motor cortex excitability were measured before and after each training type. During the passive training session, each passive movement was followed by the presentation of a playedback feedback signal. Other training parameters were kept constant, including concentration, using a previously described electroencephalogram (EEG)-modulation task (modified from Ref. 10). Performance improvements were measured as the increase in the number of hits within the critical temporal window. Wrist flexion movements were only possible in the desired axis (flexion extension). Failure to execute a full motion resulted in a failed trial. The temporal features of each movement were monitored and feedback was provided. The main finding of the study was that active training resulted in clear performance 
improvements in the motor task, whereas passive training did not. Resting motor thresholds (rMT), recruitment curves (RC), and intracortical inhibition and facilitation (ICF) were measured before and after each intervention from extensor carpi radialis muscle. Three separate analysis of variances for $\mathrm{rMT}, \mathrm{RC}$, and ICF with factors training (active/passive) and time (pre/post), revealed no changes in rMT but an increase of RC and ICF after active but not after passive training. fMRI revealed an increase of the activated cluster within the precentral and postcentral gyrus after active training. Therefore, active training resulted in more prominent performance improvements, accompanied by increased processing and motor cortical excitability within M1. Changes in ICF are consistent with the involvement of glutamatergic neurotransmission mechanisms. ${ }^{11}$ All together, these findings support the concept of a pivotal role of voluntary drive in motor learning.

In another experiment, Kaelin-Lang et al $^{12}$ investigated the influence of voluntary and passively elicited thumb movements on encoding of an elementary motor memory in the primary motor cortex. In this experimental protocol, a group of healthy volunteers underwent a period of 30 minutes training consisting of performance of (a) voluntary thumb movements and (b) passively elicited movements, all performed at $1 \mathrm{~Hz}$ in different sessions randomly ordered. The purpose was to determine to what extent each training strategy generated a directional bias in TMS-evoked movements, a measure of encoding of an elementary motor memory in the primary motor cortex, ${ }^{13,14}$ that reflects the kinematic details of the practiced movements and may contribute to skill acquisition. ${ }^{13}$ Thumb movements were recorded with a 2-dimensional accelerometer mounted on the proximal phalanx of the thumb and TMS was delivered to the optimal scalp position to elicit thumb movements in a consistent direction (60 TMS stimuli at $0.1 \mathrm{~Hz}$ before and after training). The baseline TMS-evoked thumb movement direction was defined for each subject and session before training. ${ }^{14,15}$ In the active training session, subjects practiced voluntary, brisk thumb movements paced by an acoustic signal in a direction opposite to the baseline TMS-evoked thumb movement direction for 30 minutes $\left(1 \mathrm{~Hz}^{13}\right)$. After each voluntary movement, the thumb returned to the start position by relaxation, as confirmed by electromyogram (EMG) monitoring. Monitoring accuracy and consistency of training was carried out online using the acceleration signal. In the passive training session, the same experimenter moved the subject's thumb passively and briskly in a direction opposite to the baseline TMS-evoked movement direction for 30 minutes $(1 \mathrm{~Hz})$. Each passive movement was paired with presentation of the same acoustic signal as in the active training session. To describe the training effects on TMS-evoked movement directions, a training target zone as a window of \pm 20 degrees centered on the training direction was defined. ${ }^{14,15}$ The end point measure of this study was the increase in the proportion of TMS-evoked movements that fell within the training target zone after training. The main result of this study was that active motor training led to encoding of a motor memory in the primary motor cortex, whereas passive training did not. Additionally, active training led to a differential modulation of corticomotor excitability, enhanced in muscles agonistic to the training motions and depressed in muscles antagonistic to the training motions, possibly reflecting the neurophysiologic correlates of ${ }^{14}$ or contributes to ${ }^{16}$ the newly encoded motor memory.

To what extent these findings in healthy volunteers impact on clinical neurorehabilitation remains to be determined. However, they suggest that the use of passive training strategies should not replace active motor training in able individuals. On the other hand, it is possible that passive training strategies may play a more prominent role in individuals unable to perform voluntary motions during rehabilitative treatments, an issue that deserves further investigation.

Two recent studies used passive training protocols in moderately affected stroke patients. Hesse et $\mathrm{al}^{17}$ used bilateral robot-assisted repetitive motor training for 15 minutes a day over a period of 3 weeks in 12 chronic hemiparetic patients. They did not observe significant functional improvements of motor performance as assessed with the Rivermead Motor Scale but a significant decrease of spasticity of wrist and finger joints. In another study, Lindberg et al $^{18}$ combined passive and active training components in 10 chronic stroke patients who showed mild to severe functional impairment in the affected upper limb. Training lasted 4 weeks, was performed 4 times a week, and included active warm-up (5 to $10 \mathrm{~min}$ ) and stretching (for patients with increased muscle tone; $5 \mathrm{~min}$ ), repetitive passive movements guided by a physiotherapist in a functional movement pattern (reaching, grasping for $20 \mathrm{~min}$ ), and active training ( 5 min, mimicking the passively guided movements). During passive training, subjects were instructed to "observe and feel" the movement, probably activating additional neural networks involved in motor imagery and action observation. The authors reported an improvement in range of motion and Motor Assessment Scale.

\section{IMAGERY IN NEUROREHABILITATION AND MOTOR CONTROL}

\section{Motor Imagery}

Motor imagery activates cortical regions that overlap with those activated during motor activity. For instance, fMRI studies investigating imagery of finger and hand movements ${ }^{19-22}$ demonstrated activation of the supplementary motor area (SMA), the premotor cortex (PMC), and the cerebellum but also the primary motor cortex contralateral (cM1) to the imagined movements. fMRI and TMS studies ${ }^{23}$ demonstrated that $\mathrm{cM} 1$ is activated during imagery tasks of increasingly complex movements, a result consistent with a previous finding of more prominent involvement of $\mathrm{M} 1$ with performance of complex motor sequences. ${ }^{24}$ Further evidence for the 
existence of common brain regions engaged in performance and imagination of movements comes from work by $\mathrm{Li}$ et al. ${ }^{25}$ These authors illustrated similarities in characteristics of finger interactions during both motor imagery and motor execution. Although motor performance and motor imagery activate similar neural networks, the psychologic setting in place clearly differs. According to Jeannerod ${ }^{26}$ motor imagery is accomplished by the conscious engagement of brain regions usually activated unconsciously during movement preparation. Interestingly, motor imagery does not require the presence of a limb. For example, patients with traumatic limb amputation ${ }^{27}$ or with deafferentation after complete thoracic spinal cord injury ${ }^{28}$ can perform imagery tasks involving the missing/deafferented body part. These processes are likely to involve body part representations within the sensorimotor areas of the brain that remain "linked" in some way to the missing/deafferented body part even years after the event. ${ }^{29}$ Therefore, the process of imagination is not dependent on the ability to execute a movement but rather on central processing mechanisms. As such it is conceivable that training using imagery could facilitate the organization of central motor commands.

\section{Mental Practice}

Physical and imagery motor training, for example, during performance of sequential foot movements, are associated with activation in overlapping brain regions including the inferior left parietal lobe and the left cerebellar hemispheres. ${ }^{30}$ Additional cortical sites seem to be particularly relevant for motor imagery. For example, patients with parietal ${ }^{31}$ and left lateral prefrontal $^{32}$ lesions are less able to imagine themselves performing a motor task. Patients with putaminal lesions exhibit selective impairment in kinesthetic but not visual imagery, ${ }^{33}$ pointing to the role of subcortical structures in motor imagery. Overall, there are several common nodes in the cortico-subcortical networks activated by motor imagery and execution.

These results supported previous empirical findings, leading to the proposal that practice of motor imagery techniques could contribute to neurorehabilitative efforts, particularly in patients unable to perform motor training because of weakness. Support for this proposal comes from the documented beneficial effects of mental practice, a form of motor imagery, on motor performance in some athletes $^{34}$ and musicians. ${ }^{35}$ Roure et $\mathrm{al}^{36}$ showed that athletes with best imagery capacity during training tasks were the ones who improved most in their volleyball performance. In healthy volunteers, performance and imagery for 5 days of a complex finger motor sequence led to characteristic increases in motor cortical excitability of the long finger flexors/extensor muscles involved in the tasks as assessed with TMS. ${ }^{35}$ Although subjects who performed physical training had the most prominent performance improvement, those doing motor imagery also improved to a lesser extent. An additional feature of this study was that subjects in the motor imagery group reached the same increase in motor excitability as the practice group with a second imagery session. Training in healthy volunteers using imagery techniques over a 4-week period results in improvements in performance of isometric movements (motor execution: improvements of $30 \%$; imagery: improvements of $22 \%$ ) and movement trajectories. ${ }^{37-39}$ The magnitude of improvement with imagery training is usually lower than that accomplished with real physical training, but it should be kept in mind that physical training is not possible in certain forms of motor disability after stroke (see Ref. 40).

Motor imagination requires conscious activation of brain regions involved in movement preparation and execution accompanied by voluntary inhibition of actual movement. ${ }^{27}$ The ability of healthy humans and patients with stroke to completely inhibit motor activity during motor imagery varies, and for that reason experimental protocols should ideally include high-sensitivity polygraphic EMG and kinematic monitoring to secure relaxation (eg, Ref. 30).

It has been proposed ${ }^{40}$ that "... mental practice with motor imagery requires that subjects have all the necessary declarative knowledge about the different components of the task before practicing. However, as with physical practice, rehearsing of the task with motor imagery can also give access to non conscious processes involved in learning the skilled behavior." Jackson et $\mathrm{al}^{40}$ concluded that "internally driven images which promote the kinesthetical feeling of movements would best activate the different non conscious processes involved during motor task training." The fact that only highly specialized athletes ${ }^{34}$ and musicians ${ }^{35}$ apply imagery techniques for training underlines the importance of a high level of proficiency in performance of the motor task for the successful use of imagery. Jeannerod ${ }^{26}$ highlighted the role of a preceding execution for a vivid kinesthetic image. Under this view, imagery training may represent a complementary or adjuvant technique to voluntary motor training but may not replace it. This concept seems to apply to patients with motor deficits secondary to lesions of the central nervous system, who have kinesthetic memories of themselves performing activities of daily living with the affected limbs. It is likely that in these cases, motor imagery could enforce the beneficial effects of customarily used motor training protocols, perhaps retrieving these images into more accessible working memory storage. ${ }^{41}$

For example, motor imagery training could start early in the rehabilitative process, when motor execution training is still not possible because of excessive weakness. It would be desirable to design experimental protocols to determine if such intervention could speed up recovery of function, perhaps by accelerating the inclusion of physical training protocols in the rehabilitative schedule. Previous studies of motor imagery in stroke (eg, Refs. 42, 43) had strict inclusion criteria accepting patients with low neuropsychologic impairment, high imagery scores and predominantly at the chronic stage. One study compared conventional physiotherapy and physiotherapy plus motor imagery in subacute with chronic stroke patients 
and demonstrated a greater improvement of hand function with the additional mental practice. ${ }^{44}$ Performance improvements demonstrated with motor imagery alone after chronic stroke, as expected, were more modest than those identified with physical training.

At least 2 imagery techniques have been differentiated. In 1, a visual representation of the moving $\operatorname{limb}$ is generated where the subject is a spectator of the movement (external imagery). ${ }^{45}$ In the other, a kinesthetic feeling of the movement is created, where the subject mentally simulates him or herself performing the task (internal imagery). ${ }^{45}$ For motor training purposes, kinesthetic imagery has been favored. ${ }^{40}$

It is conceivable that a combination of different strategies could enhance rehabilitative efforts. For example, the instruction to imagine performance of hand movements in a stroke patient may be accompanied by passively induced hand movements to provide corresponding somatosensory input ${ }^{43,46}$ or/and by observation of another individual performing the same task. ${ }^{41}$ Observation of movements and provision of somatosensory feedback in synchrony with motor imagery could potentially have facilitatory effects through Hebbian mechanisms on motor executive areas in these patients. ${ }^{47}$

\section{POSSIBLE SUBSTRATES UNDERLYING SIMPLE AND COMPLEX MOVEMENTS AFTER STROKE}

There are well-documented differences between the neural substrates underlying the performance of motor tasks of different complexities. In general, the more complex the motor action, the broader the activity of related neuronal nets. Complex movements activate more extensive regions in the SMA, the dorsal PMC, and the ipsilateral and contralateral cerebellar hemispheres than simple movements. ${ }^{24,48}$ As healthy volunteers practice a complex motor task, performance becomes more accurate and often automatic, resulting in a sharpening of EMG patterns and more precise coordination. ${ }^{49}$ Associated movements of the other hand, often present during early performance of a complex unilateral motor sequence, tend to disappear or improve over time. ${ }^{50}$ Such behavioral modifications that accompany motor skill acquisition and long-term practice effects are accompanied by structural and functional changes within the brain. ${ }^{51,52}$ The neural substrates underlying performance of complex motor sequences tends to shrink with training both in normal volunteers ${ }^{52}$ and in patients with stroke underlying rehabilitative treatments. ${ }^{53}$ Consistent with these findings, professional pianists performing complex finger motor sequences show decreased motor activation with less contribution of the SMA, PMC, and the ipsilateral primary motor cortex relative to nonmusicians. ${ }^{54,55}$ These authors proposed that prolonged musical training and efficiency in playing an instrument is associated with the assignment of neural resources to other aspects of artistic and motor performance. An alternative interpretation is that with practice, an economy principle applies and the activated brain regions become more proficient in performing the task. Other investigators reported enlargement of cortical activation during learning (and repetition) of rapid finger movement sequences over weeks. $^{56,57}$ Seitz et al $^{58}$ reported that $\mathrm{cM} 1$ and bilateral PMC were more intensely activated as movement trajectories become increasingly well rehearsed. Along a similar line, it has been shown that professional violinists exhibit a use-dependent enlargement of cortical somatosensory representation of left-hand digits. ${ }^{59}$ Clearly, more work is required to understand reorganizational changes associated with practice of complex motor tasks. One interesting finding is that a decrease in cerebellar activation after continued practice is followed by an increase of activation within the basal ganglia. ${ }^{60}$ These findings raised the hypothesis that early motor learning may rely predominantly on activity in the cerebellocortical network, whereas automatization may rely more on striato-cortical circuits. ${ }^{61}$

A recent study compared activation maps of professional and amateur violinists during actual performance of the first 16 bars of Mozart's violin concerto in $G$ major (KV216). Activation in professional musicians increased in the contralateral primary sensorimotor cortex and the ipsilateral anterior cerebellar hemisphere ${ }^{62}$ and decreased in the SMA, the bilateral PMC, and the contralateral cerebellar hemisphere relative to nonprofessional musicians. These changes occurred in the setting of increased EMG activity from finger extensors in the professional relative to the amateur group. The magnitude of EMG activity at the target muscles during performance of a musical piece correlated with the training time with the instrument. ${ }^{63}$ The increased ipsilateral M1 activation, present during the early phase of motor learning, ${ }^{64}$ may also be explained by additional associated movements of the right hand in less trained subjects, ${ }^{50}$ underlining the need for careful EMG and motor kinematics monitoring.

This information is relevant to the understanding of the neural substrates underlying recovery of function in patients with stroke. An initial observation was that chronic stroke patients performing simple movements show activation patterns that resemble those of healthy volunteers performing complex motor sequences. ${ }^{65}$ It is possible that the larger brain regions activated in the patient group with simple movements are a consequence of increased task difficulty. An additional issue that remains to be investigated is the specific role of extensive activation areas in stroke patients, particularly because there is an inverse correlation between the magnitude of activation in chronic stroke patients with capsula interna infarcts and the degree of motor recovery. ${ }^{53} \mathrm{~A}$ shift of patterns of lesion-induced reorganization in chronic stroke patients have been observed towards a more normal pattern in those patients with good recovery. ${ }^{53,66}$

In summary, functional changes in cerebral activity seen after active motor training, motor imagery, and training in complex movements have been described which correlate with motor performance in healthy 
subjects and patients with chronic stroke. Different strategies including motor imagery, action observation, attentional focusing, and motivation as well as pharmacologica $^{67}$ and brain stimulation ${ }^{68}$ tools could enhance the beneficial effects of motor training on neurorehabilitation. Understanding of the functional role of various neuroimaging activation patterns could further enhance the development of adjuvant rehabilitative tools.

\section{REFERENCES}

1. Liepert J, Graef S, Uhde I, et al. Treatment-induced cortical reorganization after stroke in humans. Stroke. 2000;31:1210-1216.

2. Ward NS, Brown MM, Thompson AJ, et al. Neural correlates of outcome after stroke: a cross-sectional fMRI study. Brain. 2003; 126:1430-1448.

3. Johansen-Berg H, Dawes H, Guy C, et al. Correlation between motor improvements and altered fMRI activity after rehabilitative therapy. Brain. 2002;125:2731-2742.

4. Schaechter JD, Kraft E, Hilliard TS, et al. Motor recovery and cortical reorganization after constraint-induced movement therapy in stroke patients: a preliminary study. Neurorehabil Neural Repair. 2002;16:326-338.

5. Rossini PM, Calautti C, Pauri F, et al. Post-stroke plastic reorganisation in the adult brain. Lancet Neurol. 2003;2:493-502.

6. Calautti C, Baron JC. Functional neuroimaging studies of motor recovery after stroke in adults: a review. Stroke. 2003;34:1553-1566.

7. Alary F, Doyon B, Loubinoux I, et al. Event-related potentials elicited by passive movements in humans: characterization, source analysis, and comparison to fMRI. Neuroimage. 1998;8:377-390.

8. Carel C, Loubinoux I, Boulanouar K, et al. Neural substrate for the effects of passive training on sensorimotor cortical representation: a study with functional magnetic resonance imaging in healthy subjects. J Cereb Blood Flow Metab. 2000;20:478-484.

9. Lotze M, Braun C, Birbaumer N, et al. Motor learning elicited by voluntary drive. Brain. 2003;126:866-872.

10. Birbaumer N, Ghanayim N, Hinterberger T, et al. A spelling device for the paralysed. Nature. 1999;398:297-298.

11. Ziemann U, Hallett M, Cohen LG. Mechanisms of deafferentationinduced plasticity in human motor cortex. J Neurosci. 1998;18: 7000-7007.

12. Kaelin-Lang A, Sawaki L, Cohen LG. Role of voluntary drive in encoding an elementary motor memory. J Neurophysiol. 2004;93: 1099-1103.

13. Classen J, Liepert A, Wise SP, et al. Rapid plasticity of human cortical movement representation induced by practice. $J$ Neurophysiol. 1998;79:1117-1123.

14. Butefisch CM, Davis BC, Wise SP, et al. Mechanisms of usedependent plasticity in the human motor cortex. Proc Natl Acad Sci USA. 2000;97:3661-3665.

15. Sawaki L, Boroojerdi B, Kaelin-Lang A, et al. Cholinergic influences on use-dependent plasticity. J Neurophysiol. 2002;87: 166-171.

16. Floel A, Breitenstein C, Hummel F, et al. Dopaminergic influences on formation of a motor memory. Ann Neurol. 2005;58:121-130.

17. Hesse S, Schulte-Tigges G, Konrad M, et al. Robot-assisted arm trainer for the passive and active practice of bilateral forearm and wrist movements in hemiparetic subjects. Arch Phys Med Rehabil. 2003;84:915-920.

18. Lindberg P, Schmitz C, Forssberg H, et al. Effects of passive-active movement training on upper limb motor function and cortical activation in chronic patients with stroke: a pilot study. $J$ Rehabil Med. 2004;36:117-123.

19. Leonardo M, Fieldman J, Sadato N, et al. A functional resonance imaging study of cortical regions associated with motor task execution and motor ideation in humans. Hum Brain Mapping. 1995;3:83-92.

20. Sabbah P, Simond G, Levrier O, et al. Functional magnetic resonance imaging at $1.5 \mathrm{~T}$ during sensory motor and cognitive tasks. Eur Neurol. 1995;35:131-136.
21. Porro CA, Francescato MP, Cettolo V, et al. Primary motor and sensory cortex activation during motor performance and motor imagery: a functional magnetic resonance imaging study. $J$ Neurosci. 1996;16:7688-7698.

22. Lotze M, Montoya P, Erb M, et al. Activation of cortical and cerebellar motor areas during executed and imagined hand movements: An fMRI study. J Cogn Neurosci. 1999;11:491-501.

23. Kuhtz-Buschbeck JP, Mahnkopf C, Holzknecht C, et al. Effectorindependent representations of simple and complex imagined finger movements: a combined fMRI and TMS study. Eur J Neurosci. 2003;18:3375-3387.

24. Gerloff C, Corwell B, Chen R, et al. The role of the human motor cortex in the control of complex and simple finger movement sequences. Brain. 1998;121:1695-1709.

25. Li S, Kamper DG, Stevens JA, et al. The effect of motor imagery on spinal segmental excitability. J Neurosci. 2004;12:9674-9680.

26. Jeannerod $M$. The representing brain: neural correlates of motor intention and imagery. Brain Behav Sci. 1994;17:187-245.

27. Schilder P. The Image and Appearance of the Human Body. Milton Park, UK: Routledge \& Kegan Paul; 1935.

28. Decety J, Boisson D. Effect of brain and spinal cord injuries on motor imagery. Eur Arch Psychiatry Clin Neurosci. 1990;240:39-43.

29. Cohen LG, Topka H, Cole R, et al. Leg paresthesias induced by magnetic brain stimulation in patients with thoracic spinal cord injury. Neurology. 1991;41:1283-1287.

30. Lafleur MF, Jackson PL, Malouin F, et al. Motor learning produces parallel dynamic functional changes during the execution and imagination of sequential foot movements. Neuroimage. 2002; 16:142-157.

31. Sirigu A, Duhamel JR, Cohen L. The mental representation of hand movements after parietal cortex damage. Science. 1996;273: $1564-1568$

32. Johnson SH. Imagining the impossible: intact motor representations in hemiplegics. Neuroreport. 2000;11:729-732.

33. Li CR. Impairment of motor imagery in putamen lesions in humans. Neurosci Lett. 2000;287:13-16.

34. Driskell JE, Copper C, Moran A. Does mental imagery enhance performance? J Appl Psychol. 1994;79:481-492.

35. Pascual Leone A, Dang N, Cohen LG, et al. Modulation of motor responses evoked by transcranial magnetic stimulation during the acquisition of new fine motor skills. J Neurophysiol. 1995;74: 1034-1045.

36. Roure R, Collet C, Deschaumes-Molinaro C, et al. Imagery quality estimated by autonomic response is correlated to sporting performance enhancement. Physiol Behav. 1999;66:63-72.

37. Yue G, Cole KJ. Strength increases from the motor program: comparison of training with maximal voluntary and imagined muscle contractions. J Neurophysiol. 1992;67:1114-1123.

38. Yágüez L, Nagel D, Hoffman H, et al. A mental route to motor learning: improving trajectorial kinematics through imagery training. Behav Brain Res. 1998;90:95-106.

39. Gandevia SC. Mind, muscles and motorneurones. J Sci Med Sport 1999;2:167-180.

40. Jackson PL, Lafleur MF, Malouin F, et al. Potential role of mental practice using motor imagery in neurologic rehabilitation. Arch Phys Med Rehabil. 2001;82:1133-1141.

41. Stefan K, Cohen LG, Duque J, et al. Formation of a motor memory by action observation. $J$ Neurosci. 2005;25:9339-9346.

42. Weiss T, Hansen E, Rost R, et al. Mental practice of motor skills used in poststroke rehabilitation has own effects on central nervous activation. Int J Neurosci. 1994;78:157-166.

43. Miltner R, Simon U, Netz J, et al. Motor imagery in the therapy of patients with central motor deficit. Neurol Rehabil. 1999;5:66-72.

44. Page SJ, Levine P, Sisto S, et al. A randomized efficacy and feasibility study of imagery in acute stroke. Clin Rehabil. 2001; 15:233-240.

45. Solodkin A, Hlustik P, Chen EE, et al. Fine modulation in network activation during motor execution and motor imagery. Cereb Cortex. 2004;14:1246-1255.

46. Stevens JA, Stoykov ME. Using motor imagery in the rehabilitation of hemiparesis. Arch Phys Med Rehabil. 2003;84:1090-1092. 
47. Hebb DO. The Organization of Behavior: A Neuropsychological Theory. New York: Wiley; 1949.

48. Lotze M, Erb M, Flor H, et al. fMRI-evaluation of somatotopic representation in human primary motor cortex. Neuroimage. $2000 ; 11: 473-481$.

49. Seitz RJ, Roland PE. Learning of sequential finger movements in man: a combined kinematic and PET Study. Eur $J$ Neurosci. 1992;4:154-165.

50. Rijntjes M, Krams M, Müller S, et al. Associated movements after stroke. Neurol Rehabil. 1999;5:15-18.

51. Kelso JA, Fuchs A. Self-organizing dynamics of the human brain: critical instabilities and Sil'nikov chaos. Chaos. 1995;5:64-69.

52. Morgen K, Kadom N, Sawaki L, et al. Kinematic specificity of cortical reorganization associated with motor training. Neuroimage. 2004;21:1182-1187.

53. Ward NS. Functional reorganization of the cerebral motor system after stroke. Curr Opin Neurol. 2004;17:725-730.

54. Hund-Georgiadis M, von Cramon DY. Motor learning related changes in piano players and non-musicians revealed by functional magnetic-resonance imaging. Exp Brain Res. 1999;125:417-425.

55. Jaencke L, Shah NJ, Peters M. Cortical activation in primary and secondary motor areas for complex bimanual movements in professional pianists. Cogn Brain Res. 2000;10:177-183.

56. Karni A, Meyer G, Jezzard P, et al. Functional MRI evidence for adult motor cortex plasticity during motor skill learning. Nature. 1995;377:155-158.

57. Karni A, Meyer G, Rey-Hipolito C, et al. The acquisition of skilled motor performance: fast and slow experience-driven changes in primary motor cortex. Proc Natl Acad Sci USA. 1998;95:861-868.
58. Seitz RJ, Canavan AG, Yaguez L, et al. Successive roles of the cerebellum and premotor cortices in trajectorial learning. Neuroreport. 1994;5:2541-2544.

59. Elbert $\mathrm{T}$, Pantev $\mathrm{Ch}$, Wienbruch $\mathrm{Ch}$, et al. Increased cortical representation of the fingers of the left hand in string players. Science. 1995;270:305-307.

60. Doyon J, Owen AM, Petrides M, et al. Functional anatomy of visuomotor skill learning in human subjects examined with positron emission tomography. Eur J Neurosci. 1996;8:637-648.

61. Doyon J, Song AW, Karni A, et al. Experience-dependent changes in cerebellar contributions to motor sequence learning. Proc Natl Acad Sci USA. 2002;99:1017-1022.

62. Lotze M, Scheler G, Tan HRM, et al. The musician's brain: functional imaging of amateurs and professionals during performance and imagery. Neuroimage. 2003;20:1817-1829.

63. Sloboda JA. Individual differences in music performance. TICS 2000;4:397-403.

64. Andres FG, Mima T, Schulman AE, et al. Functional coupling of human cortical sensorimotor areas during bimanual skill acquisition. Brain. 1999;122:855-870.

65. Weiller C. Imaging recovery from stroke. Exp Brain Res. 1998; 123:13-17.

66. Calutti C, Baron JC. Functional neuroimaging studies of motor recovery after stroke in adults: a review. Stroke. 2003;34:1553-1566.

67. Knecht S, Breitenstein C, Bushuven S, et al. Levadopa: faster and better word learning in normal humans. Ann Neurol. 2004;56:20-26.

68. Hummel F, Celnik P, Giraux P, et al. Effects of non-invasive cortical stimulation on skilled motor function in chronic stroke. Brain. $2005 ; 128: 490-499$ 